\title{
Diagnostic difficulties in Krabbe disease: a report of two cases and review of literature
}

\author{
Krystyna Szymańska ${ }^{1,2}$, Agnieszka Ługowska ${ }^{3}$, Milena Laure-Kamionowska ${ }^{1}$, Monika Bekiesińska-Figatowska \\ Dorota Gieruszczak-Białek ${ }^{5}$, Małgorzata Musielak³, Sabrina Eichler ${ }^{6}$, Anne-Katrin Giese ${ }^{7}$, Arndt Rolfs ${ }^{6,7}$ \\ 1Department of Experimental and Clinical Neuropathology, Mossakowski Medical Research Centre, Polish Academy of Sciences, \\ Warsaw, Poland, 2Department of Child Psychiatry, Medical University of Warsaw, Poland, 3Institute of Psychiatry and Neurology, \\ Department of Genetics, Warsaw, Poland, ${ }^{4}$ Department of Diagnostic Imaging, Institute of Mother and Child, Warsaw, Poland, \\ ${ }^{5}$ Department of Pediatrics, Medical University of Warsaw, Poland, ${ }^{6}$ Centogene AG, Rostock, Germany, ${ }^{7}$ Albrecht-Kossel-Institute for \\ Neuroregeneration, University of Rostock, Germany
}

\begin{abstract}
Globoid cell leukodystrophy (GLD, also known as Krabbe disease), whose pathophysiology is still not completely elucidated, is an inherited, metabolic, and neurodegenerative disease, caused by the deficiency of $\beta$-galactocerebrosidase (GALC) or in very rare cases by lack of active saposin $A$.

We describe two patients, in whom first MRI changes were not suggestive of GLD. Additionally, in Patient 1, the residual $\beta$-galactocerebrosidase activity was rather high leading to difficulties in the diagnosing process. Molecular analysis of the GALC and PSAP genes in Patient 1, and of the GALC gene in Patient 2 confirmed the diagnosis of Krabbe disease. We have detected a novel mutation in the GALC gene in Patient 2, a deletion in exon 16, leading to the STOP codon (c.1851delT, p.Y617X). This deletion interrupts the reading frame prematurely: codon 617 is replaced by a STOP codon. A careful clinical description of presented patients is followed by a discussion of radiological, biochemical, genetic, and neuropathological studies. It concludes with a discussion of the potential difficulties encountered when diagnosing patients with rare diseases. In Patient 1 the postmortem examination of CNS revealed the presence of globoid cells grouped in multiple clusters seen in the white matter near the vessels.

We would like to emphasize that proper clinical-radiological-biochemical co-operation and exchange of information between parents and specialists is a key issue in the diagnosis of rare and difficult neurological diseases, in particular, if the clinical picture is inconclusive.
\end{abstract}

Key words: Krabbe disease, globoid cells leukodystrophy, $\beta$-galactocerebrosidase, psychosine, demyelination, lysosomal disorder.

\section{Introduction}

Globoid cell leukodystrophy (GLD, also known as Krabbe disease; OMIM 245 200), is an inherited, meta- bolic, and neurodegenerative disease. It was described for the first time by Knud Krabbe, a Danish neurologist, in 1916, in two sibs ("A new familial infantile form of diffuse brain sclerosis", Brain, 1916) [14]. A charac- 
teristic, histologic feature of this disorder is the presence of globoid cells in the nervous system. In 1970, the laboratory of Kunihiko Suzuki identified genetic deficiency of $\beta$-galactocerebrosidase as the biochemical cause of Krabbe disease [22]. These authors also found that the composition of the myelin sheath in affected and healthy individuals does not differ and there is no overt accumulation of galactosylceramide in the brain of patients with Krabbe disease [6]. This distinguishes Krabbe disease from other lysosomal diseases, including metachromatic leukodystrophy, in which, though metabolically closely related, the accumulation of the substrate is present. Two years later in the same laboratory, the "psychosine hypothesis" was formulated. This hypothesis explains the observed findings and despite some actual changes, is in essence still valid [19].

Krabbe disease can also be caused by the absence of a physiologically active sphingolipid activator protein - saposin A (SAP-A).

The infantile classic form of GLD is a severe, rapidly progressing, demyelinating disease. First symptoms appear before the age of 6 months and they include hyper-irritability, stiffness, hyperactive reflexes, episodes of elevated temperature followed by psychomotor deterioration, seizures, spasticity, and vision loss. In late-onset juvenile and adult forms of GLD the clinical outcome is more variable but symptoms of weakness, vision loss, and arrest of intellectual development are always evident [30].

Magnetic resonance imaging (MRI) shows characteristic changes. In early-onset Krabbe disease periventricular white matter and corpus callosum abnormalities with relative sparing of the $U$ fibers are visible. A radiating pattern with stripes within the cerebral white matter is often observed [26]. Involvement of the brain stem and cerebellum reveals earlier than white matter involvement. In late-onset Krabbe disease the cerebellar white matter is spared. In adult form, changes can only occur in the pyramidal tract from the motor cortex to the brain stem [26].

Krabbe disease is inherited in an autosomal recessive manner. The incidence of GLD varies from 0.4 to 1.9 in 100000 live births according to the population studied $[10,24]$.

The GALC gene coding for the $\beta$-galactocerebrosidase protein is located on chromosome $14 q 31$ and encompasses 17 exons. More than 110 disease-causing mutations have been identified in the GALC gene.
The $30 \mathrm{~kb}$ deletion covering exons $11-17$ is responsible for about $45 \%$ of mutated alleles in patients of European origin with Krabbe disease. In homozygosity or in heterozygosity, together with another severe mutation, it leads to the classic infantile form of GLD. Mutation c.857G >A (p.Gly286Asp in exon 8) in a homoor heterozygous state, together with another mutation, always results in late-onset GLD. In general, it is difficult to predict the clinical picture based on the type or location of missense mutation [31]. Four common polymorphisms in the GALC gene are known, resulting in pseudodeficiency of $\beta$-galactocerebrosidase activity.

The native, $80 \mathrm{kDa}$ precursor protein of $\beta$-galactocerebrosidase (GALC; EC 3.2.1.46) is proteolytically cleaved into active subunits of $50 \mathrm{kDa}$ and $30 \mathrm{kDa}$. These subunits aggregate to form a very high molecular weight complex (over 800000 kDa). GALC is a lysosomal hydrolase that is essential for glycosphingolipid catabolism. It cuts galactose from galactocerebroside (galactosylceramide). Other GALC substrates are: galactosylsphingosine (psychosine), monogalactosyldiglyceride, and lactosylceramide (under specific conditions) [30]. For its action GALC requires the presence of sphingolipid activator protein A, saposin A.

Measurement of GALC activity in blood leukocytes or cultured skin fibroblasts is possible using the radiolabelled natural substrate galactocerebroside or artificial 6-hexadecanoylamino-4-methylumbelliferyl- $\beta$-D-galactopyranoside (6HMU-Gal). There is an overlap in GALC activity ranges between the control groups and carriers because of the multiple polymorphic changes in the GALC gene. This presents a serious diagnostic problem, demanding a careful diagnostic procedure, involving substrate-loading tests in cultured skin fibroblasts or sequencing analysis of the GALC gene.

An example of an animal model of GLD is a twitcher mouse (twi/twi), which develops most of the pathological hallmarks seen in humans. Detailed research conducted on mice has expanded our understanding of the pathogenesis of the disease.

We present here case reports of two patients with the infantile form of Krabbe disease. A clinical description, followed by a discussion of radiological, biochemical, genetic, and neuropathological studies concludes with a discussion of the potential difficulties encountered when diagnosing patients with rare diseases. 


\section{Case reports}

\section{Patient 1}

Patient 1 was a boy, the first child born at term in 2009 to probably unrelated parents. Birth weight was $3390 \mathrm{~g}$, head circumference $33 \mathrm{~cm}$, Apgar score: 10. At the age of six months, after three days of fever, he was admitted to a paediatric hospital. The parents had not noticed any alarming symptoms before, however the boy had never been seen by a paediatrician, and was vaccinated only at birth. On admission to the hospital, the boy was alternately drowsy or agitated. Abnormal posturing was observed. A lumbar puncture was performed. Pleocytosis was 3 and protein level was elevated to $293 \mathrm{mg} / \mathrm{dl}$. Treatment with an antibiotic, acyclovir, and steroids was started. After a few days the patient improved but voluntary movements deteriorated. Additional lumbar punctures revealed elevated protein levels at 246, 187, $138 \mathrm{mg} / \mathrm{dl}$. An electroencephalogram showed asymmetry of background activity. A fundus examination was normal. An ultrasound examination of the brain was normal.

The first MRI of the brain revealed periventricular white matter T2-hyperintense areas, which were more pronounced than hyperintensity of physiologically unmyelinated subcortical white matter. The posterior limbs of the internal capsules as well as the cerebellar white matter were hyperintense. The hilus of the dentate nucleus was also bilaterally involved. The brain stem and the corpus callosum were intact at the time of the first examination. No contrast enhancement of the brain and meninges was found. The above description was made retrospectively. In the initial assessment of this examination, the supratentorial changes were ignored and only cerebellar involvement was noticed and attributed to encephalitis (cerebellitis).

An MRI of the spinal cord was performed and contrast enhancement of lumbosacral nerve roots and meninges along the spinal cord and on the ventral surface of the medulla oblongata was obtained.

In the search for a possible inflammatory aetiology, serological diagnostic tests towards TORCH were performed. The results remained in the normal range. Because of a family history of tuberculosis and enlarged intrathoracic lymph nodes, gastric lavages for isolation of Mycobacterium tuberculosis were performed. In one out of 6 specimens the genetic material of $M$. tuberculosis was found. However, further research in a specialized centre ruled out tuberculosis. Highly specialized laboratory analyses excluded some rare metabolic disorders. The very long chain fatty acids (VLCFA) level was normal. The activity of some lysosomal enzymes remained in the reference range: arylsulfatase A, alpha$N$-acetylgalactosaminidase, acid $\beta$-galactosidase.

On readmission to the hospital at 10 months of age, the patient displayed hyperextension of the axial muscles, worsening of voluntary movements (the upper extremities in flexion pattern, with fists; the lower extremities in a total extension with the plantar flexion), amimic face. He was noted to have a complete loss of contact.

At 13 months of age, the patient developed problems with swallowing. He had to be fed with a nasogastric tube and then by percutaneous endoscopic gastrostomy. Hypothermia $\left(35.6^{\circ} \mathrm{C}\right)$ occurred alternately with hyperthermia. Due to the progression of clinical symptoms, the next MRI was performed at that time (Fig. 1A, Fig. 2A). Periventricular changes were much more T2-hyperintense and involved larger areas of the white matter. A radiating pattern with more normal signal intensity along the perivascular spaces was observed. There were cavitary lesions in the posterior limbs of the internal capsules as well as in the posterior part of the corpus callosum. The cerebellar white matter was affected to a much larger degree than had been previously noted. Lesions in the cerebral peduncles and in the pons appeared. Cerebral and cerebellar atrophy was seen which had not been noted in the previous examination. The patient continued to deteriorate and died at 15 months of age.

Due to the deterioration of the child and the changes in neuroimaging, $\beta$-galactocerebrosidase assays were performed. The results of $\beta$-galactocerebrosidase activity measured in two different samples of blood leukocytes revealed 2.1 and $2.0 \mathrm{nmol} / \mathrm{mg}$ protein/ $18 \mathrm{hr}$, respectively (in control persons: $4-9.4 \mathrm{nmol} / \mathrm{mg}$ protein $/ 18 \mathrm{hr}$; in patients affected with Krabbe disease below $2.0 \mathrm{nmol} / \mathrm{mg}$ protein $/ 18 \mathrm{hr}$ ); in cultured skin fibroblasts: $3.7 \mathrm{nmol} / \mathrm{mg}$ protein $/ 18 \mathrm{hr}$ (in control persons 3.9-15.2). The $\beta$-galactocerebrosidase activity was analysed with the use of 6-hexadecanoylamino4-methylumbelliferyl- $\beta$-D-galactopyranoside (6-HMUgal) as a fluorogenic substrate according to the manufacturer's protocol with slight modifications (MOSCERDAM, Rotterdam, The Netherlands). Molecular genetic analysis was performed at Centogene, Germany. The PSAP and GALC genes were analysed by PCR, 

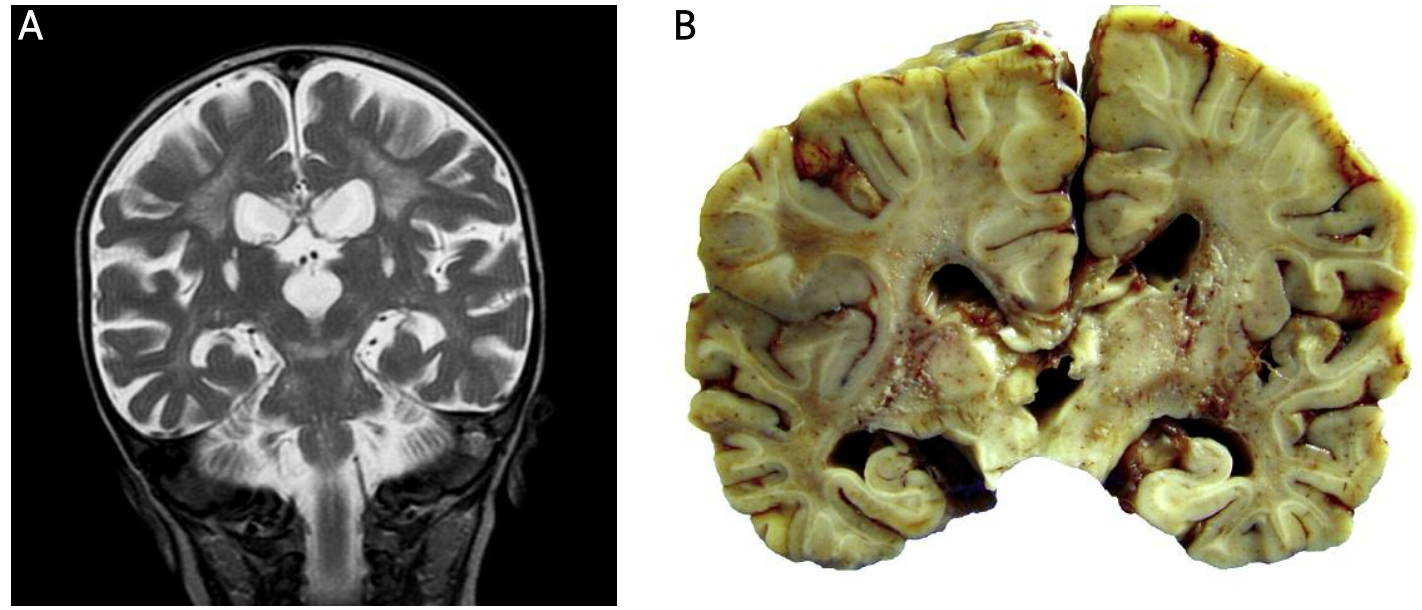

Fig. 1. Patient 1. Comparison of the MR image of the brain and neuropathological examination. A) Second $\mathrm{MRI}, \mathrm{FSE} / \mathrm{T} 2 \mathrm{WI} / \mathrm{coronal}$ plane. Radiating pattern with normal signal intensity along the perivascular spaces in the white matter of both cerebral hemispheres. The cavitary lesions in the internal capsules. Lesions in the cerebral peduncles. Cerebral and cerebellar atrophy. B) Macroscopic coronal section of the hemispheres. Damage of white matter of corona radiata. Bilateral symmetrical cavities in the posterior limb of the internal capsule and in corpus callosum. Spared U-fibers.
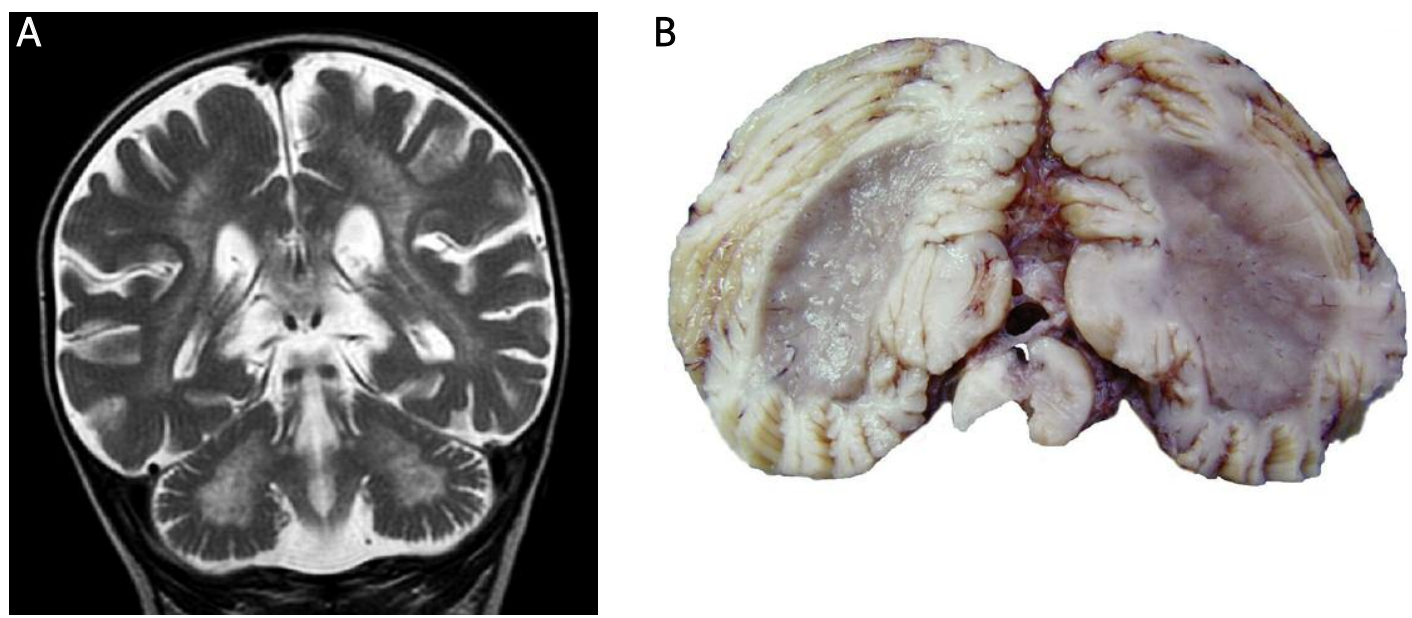

Fig. 2. Patient 1. The changes observed in the neuropathological study correspond to those seen on MRI. A) Second MRI/T2WI/coronal plane. Radiating pattern with normal signal intensity along the perivascular spaces in the white matter of the corona radiate bilaterally. Spared U-fibers. The cerebellar white matter is also symmetrically affected. Cerebral and cerebellar atrophy. B) Macroscopic coronal section of the cerebellum. Damage of the cerebellar white matter.

as well as sequencing of both DNA strands of the entire coding region and the highly conserved exon-intron splice junctions. In addition, the screening for the $30 \mathrm{~kb}$ deletion in the GALC gene was performed by PCR analysis using modified primers [15]. This analysis revealed two disease-causing mutations in a heterozygous state. We found the common $30 \mathrm{~kb}$ deletion encompassing exons 11-17 within the GALC gene. This deletion accounts for approximately $45 \%$ of the mutant alleles in individuals of European ancestry [31]. A second mutation was found in exon 11 (c.1186C $>$ T, p.R396W). This was described as disease-causing by Wenger et al. [29]. Sequencing analysis of the PSAP gene did not show any pathological mutations. 
A postmortem examination was done. The brain was fixed in formalin. The weight of the brain was $847 \mathrm{~g}$ (normal weight for this age is 944-1010 g) [7]. External appearance did not differ significantly from the norm. The optic nerves were thickened. The cerebral hemispheres turned out to be firm and rubbery. The white matter of the Corona radiata differed visibly, depending on the localization. It was relatively spared in the posterior part of the occipital lobe, greyish with linear changes in the frontal lobe and the parietal lobe. The $U$ fibers seemed to be spared. The white matter of the spinal cord was destroyed at all the levels.

The posterior limb of the internal capsule contained bilateral symmetrical cavities. The cavities were also observed in corpus callosum. The cerebellar white matter was greyish, cerebellar dentate nuclei were visible (Fig. 1B, Fig. 2B). The spinal cord was surrounded by the thickened meninges.

For light microscopy the tissue sections were embedded in paraffin. Sections were stained with hematoxylin-eosin, Luxol fast blue and cresyl violet. On light microscopic examination, multiple clusters of globoid cells in the white matter were seen. These cells were arranged in groups of a few to tens at a location near the vessels. The globoid cells differed in their number and morphology depending on the degree of disintegration of the white matter (Fig. 3). In areas with relatively well-preserved white matter, few mononuclear globoid cells were seen. In the more damaged white matter, the globoid cells were numerous, rather small, with several nuclei, located in clusters around blood vessels. In areas, where the destruction was most advanced, the globoid cells were multinucleated and contained more than 20 nuclei. In the completely disintegrated tissue, globoid cells were seen as single cells with abundant nuclei. There was intense gliosis consisting mainly of large astrocytes with ample cytoplasm. The grey matter and $U$ fibers were relatively well-preserved.

\section{Patient 2}

The second patient, a female delivered at term by caesarean section, was born in 2006 to healthy, non-consanguineous parents, without a familial history of neurodegenerative disorders. Birth weight was $3800 \mathrm{~g}$, length $55 \mathrm{~cm}$, head circumference $35 \mathrm{~cm}$, with Apgar score of 10 . In the neonatal period she was treated with an antibiotic due to green amniotic fluid aspiration. The mother, a paediatric nurse, reported to doc- tors the abnormal development of the child from the first month of life (lack of fixation, poor eye contact, weak reaction to parents, no smile in response to smiles, poor spontaneous motility). Beginning at 6 weeks of age, hypersensitivity to stimuli and episodes of opisthotonic posture were observed. An ultrasound examination of the brain and an electroencephalogram done at the age of 4 months were normal.

At 5 months of age a neurological examination demonstrated truncal hypotonia associated with significantly increased muscle tone in the upper limbs and spasticity in the lower extremities, brisk deep tendon reflexes and noticeable, bilateral Rossolimo and Babinski signs. Paucity of spontaneous limb movements and amimic face were observed in the patient. Ultrasound examination at that time revealed hyperechogenic white matter of both cerebral hemispheres with reversed cortical-subcortical echo (Fig. 4). The corpus callosum appeared to have low/normal echogenicity and normal size. The cerebellum was within normal limits.

An MRI of this patient was performed when she was 4.5 months old (Fig. 5). The posterior limbs of the internal capsules were T2-hyperintense. An unusual finding was that the white matter directly adjacent to the lateral ventricles was relatively spared except for the regions surrounding the frontal horns. The lesions in the white matter were slightly away from the lateral ventricles, without the radiating pattern, and seemed confluent with still unmyelinated subcortical white matter. Only the hili of the dentate nuclei was affected in the infratentorial compartment. There were no changes in the cerebellar white matter and in the brain stem. The corpus callosum was thin but otherwise intact. An additional finding was a thick optic chiasm and prechiasmatic sections of the optic nerves. MR spectroscopy revealed low NAA and Cr peaks, as well as a high peak of Cho and $\mathrm{ml}$ in the affected white matter.

A specialized enzymatic assay was performed, revealing very low activity of lysosomal $\beta$-galactocerebrosidase measured in blood leukocytes: $1.1 \mathrm{nmol} / \mathrm{mg}$ protein/18 hr (range: 4.0-9.4; in patients with Krabbe disease: below $2 \mathrm{nmol} / \mathrm{mg}$ protein/18 $\mathrm{hr}$ ).

Molecular analysis of the GALC gene was performed on the parents of the patient, as a DNA sample of the patient was not available. The GALC gene was analysed by PCR, as well as sequencing of both DNA strands of the entire coding region and the highly conserved exon-intron splice junctions. The reference sequence of the GALC gene is: NM_000153.2/NM_001201402.1 (a). 

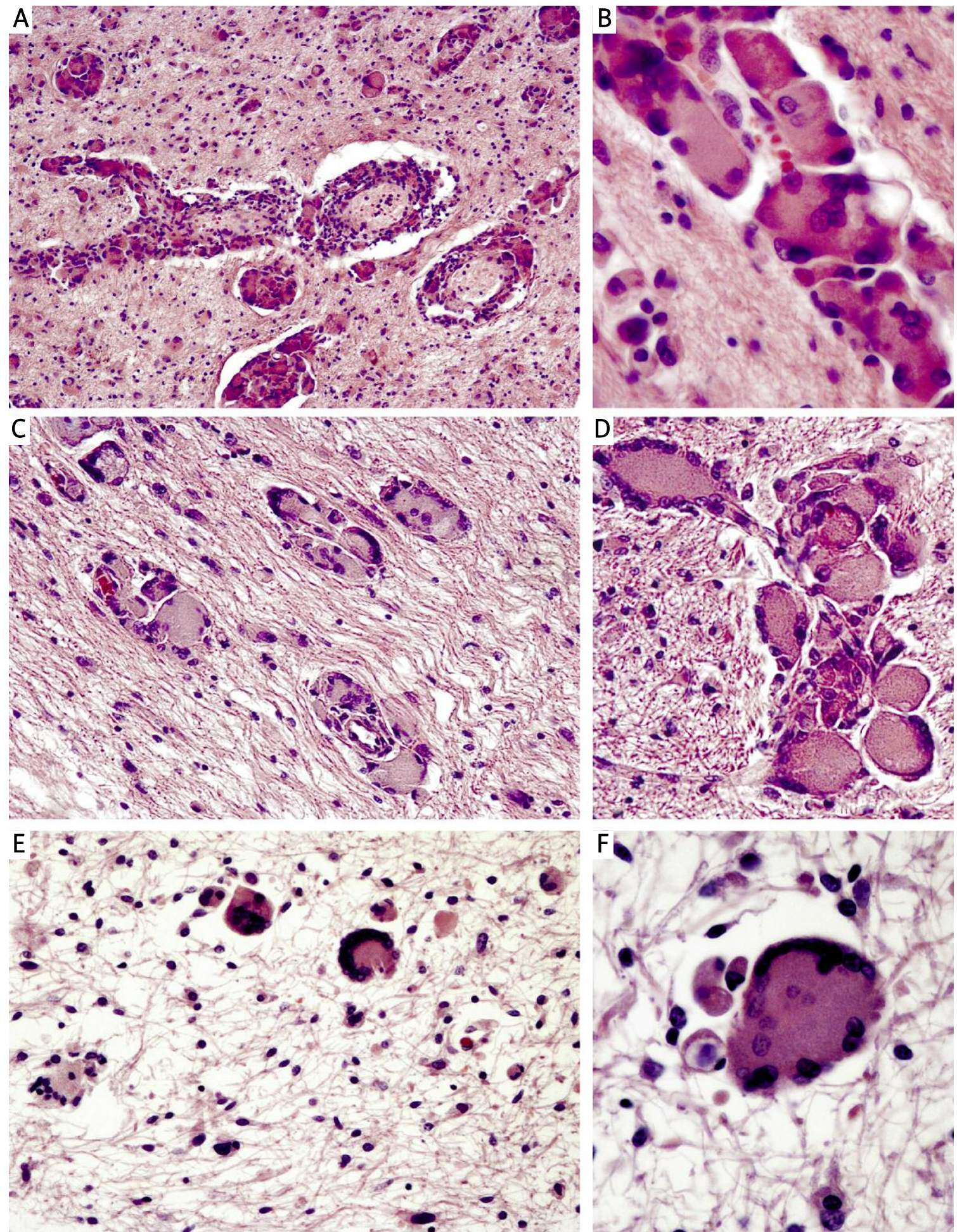

Fig. 3. Patient 1. Evaluation of globoid cells morphology changing with the degree of disintegration of the white matter. A, B) The small globoid cells located in clusters around vessels within relatively well-preserved white matter. A) Original magnification 100x; B) 400x. C, D) Numerous globoid cells, with several nuclei in the damaged white matter. C) Original magnification 100x; D) 400x. E, F) Few multinucleated globoid cells with abundant nuclei in completely disintegrated tissue. E) Original magnification 200x; F) 400x. 


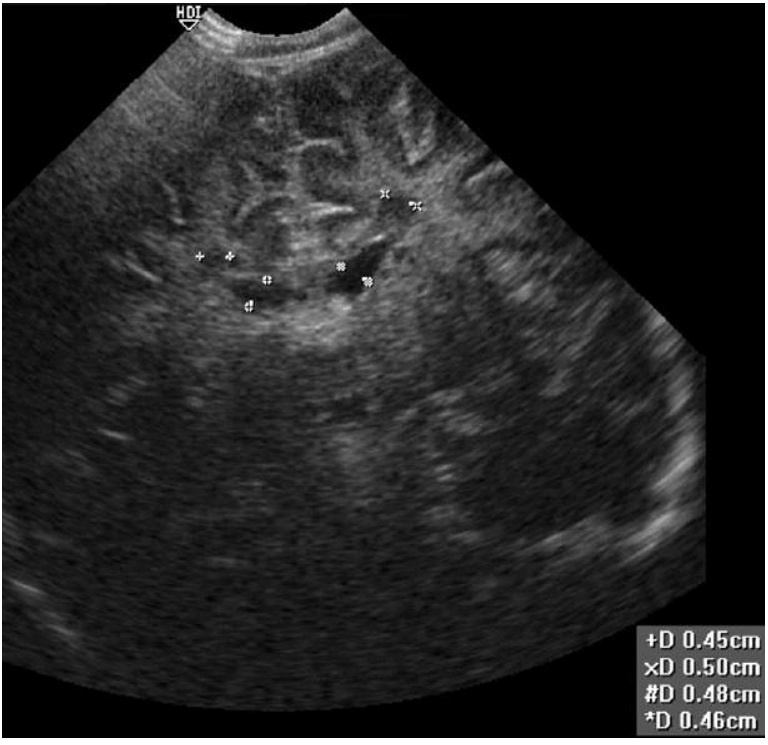

Fig. 4. Patient 2. Ultrasound examination at that time revealed hyperechogenic white matter of both cerebral hemispheres with reversed corticalsubcortical echo.

An unreported deletion in exon 16 of the GALC gene, leading to the STOP codon was identified in the patient's mother (c.1851delT, p.Y617X). This deletion interrupts the reading frame prematurely: codon 617 is replaced by a STOP codon. In the patient's father, an already described mutation p.R127X (c.379C $>T$ ) was found in exon 4 of the GALC gene [24].

\section{Discussion}

Krabbe disease is a severe, neurodegenerative disease, whose pathophysiology has still not been completely elucidated. Galactocerebroside is essential for normal function of myelin and it is almost exclusively found in the nervous system. In mice lacking the enzyme UDP-galactose ceramide galactosyltransferase (EC 2.4.1.45), which takes part in the synthesis of galactocerebroside, the myelin preserved normal ultrastructure, but galactocerebroside was replaced with glucocerebroside, a lipid absent in the myelin of healthy animals. These mice developed a neurological syndrome and showed conduction deficits in electrophysiological analysis [4].

Krabbe disease is unique among the sphingolipidoses in that the low activity of $\beta$-galactocerebrosidase does not increase the quantity of galactocerebroside in the brain. It is assumed that abnormal accumulation of psychosine (galactosylsphingosine) leads to metabolic disturbances in the cell, eventually resulting in the clinical outcome. Storage of psychosine, which is a toxic lysolipid, triggers apoptotic death of oligodendrocytes in the central and peripheral nervous systems [25].

Initially, it was thought that psychosine is generated by an alternative pathway of galactocerebroside degradation, but no additional enzymatic activity has been detected [19]. Currently, it is assumed that the metabolic generation of psychosine is possible only by
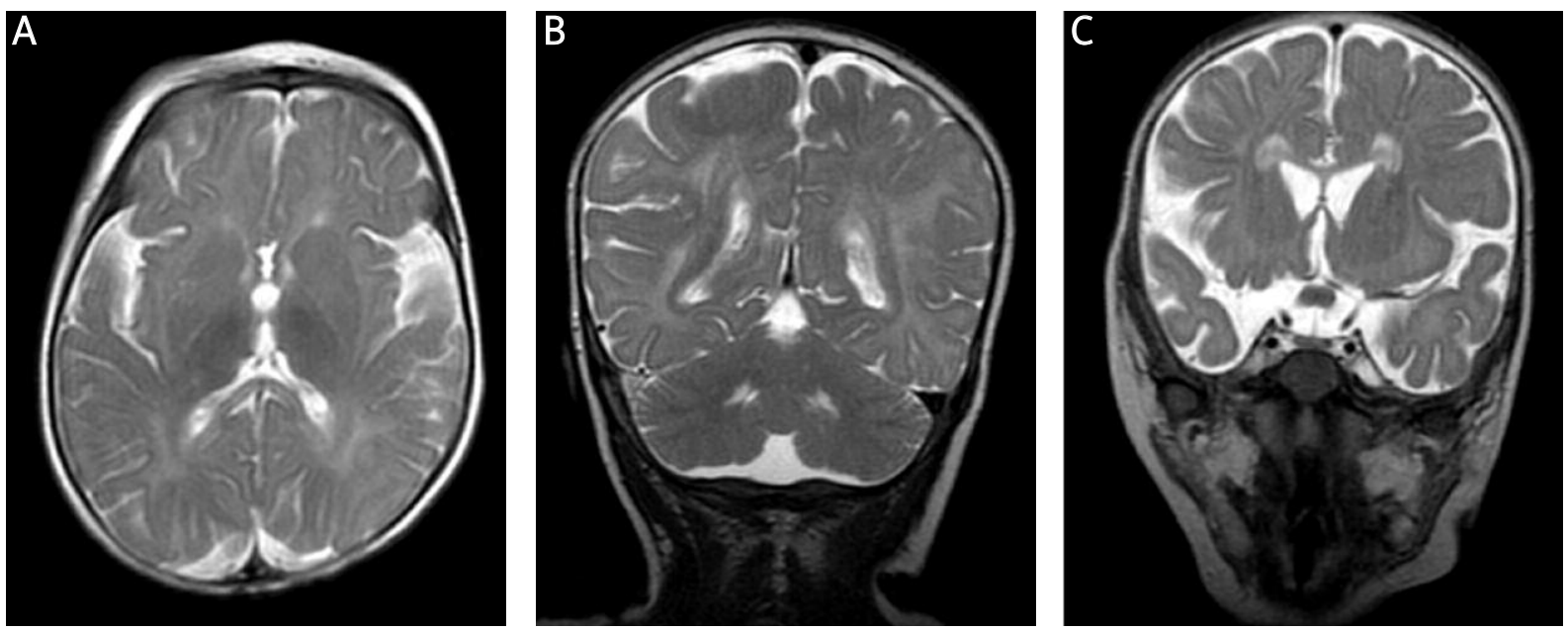

Fig. 5. Patient 2. MRI. FSE/T2WI. A) The posterior limbs of the internal capsules were T2-hyperintense.

B) The relatively spared white matter adjacent to the lateral ventricles. Affected hili of the dentate nuclei.

C) Thick optic chiasm. 
the way of a galactosylation of sphingosine $[3,23]$. A minuscule amount of psychosine is generated in a normal brain by the same enzyme that synthesizes galactocerebroside, and for this reason, most of it arises in oligodendrocytes in Krabbe disease. Neurons and astrocytes do not synthesize galactocerebroside to a significant degree, and are therefore relatively preserved, however astrocyte activation is observed. As already mentioned, psychosine is highly toxic for cells, especially for oligodendrocytes. Psychosine accumulation in the lysosomes destroys these organelles by dissolving their membrane [23]. Probably for this reason there are no enlarged lysosomes in Krabbe disease (as in other sphingolipidoses), but abnormal inclusions scattered within the cytoplasm, known to consist of galactosylceramide. Despite the lack of $\beta$-galactocerebrosidase activity there is no accumulation of galactocerebroside in the brain due to the rapid loss of oligodendrocytes, the main source of the substrate [23]. Another property of psychosine is inhibition of the last stages of the mitotic cycle during replication that induces the formation of multinucleated globoid cells [12], originating from macrophages and microglia. Kozutsumi showed that psychosine induced the formation of multinucleated cells, resulting in the reorganization of actin fibrils during cytokinesis which led to abnormal contractile movements after the formation of cleavage furrow [13].

Recently, the destructive influence of psychosine not only on oligodendrocytes, but also on axons, has been demonstrated [32]. White et al. demonstrated that twitcher mice and patients suffering from Krabbe disease stored psychosine in lipid rafts fractions obtained from cell membranes. The authors estimated the concentration of psychosine in lipid rafts and non-lipid rafts fractions of the cell membranes in twitcher mouse brain and in wild-type mouse brain at developmental stages (at 3, 20 and 40 days of age). They proved that twitcher mouse brain contained much higher levels of psychosine, from the value of seven-fold in $\mathrm{P} 3$ to $>300$ fold in P40 in comparison to the age-matched wildtype animals. This high concentration of psychosine leads to lipid rafts dysfunction and as a result drives a functional defect of signalling activity in cells [32].

Neuronal levels of psychosine are significantly lower than those found in oligodendrocytes. Castelvetri suggested that before the onset of the disease, accumulation of psychosine was below the concentration lethal for oligodendrocytes, but still sufficient to affect the structure of axons [2]. Various stages of axonal damage were described, which initiated axonal varicosities and swellings, and eventually axonal transections. The authors proved that twitcher mice are affected by an early, progressive axonopathy, operating in a dyingback pattern. It is suggested that in Krabbe disease a progressive neuronal dysfunction precedes demyelination. The authors propose a possible mechanism in which psychosine impacts the axonal function by dysregulation of axonal transport, resulting in ion imbalance and a dysfunction of axons before the myelin damage. Previous studies seem to confirm the above studies as twitcher mice treated with bone marrow transplantation and GALC-lentiviral vectors developed degeneration of axons and died with symptoms of progressive neurological degeneration despite achieving $30 \%$ of $\beta$-galactocerebrosidase activity [8]. Both of these studies may explain the therapeutic failure in Krabbe disease patients.

We report two patients suffering from the most common classic infantile form of GLD. In both presented individuals the diagnosis of GLD was delayed for various reasons. In the first case, the parents claimed that their son developed normally until the onset of fever at six months of age. Most probably, this information was the basis for further diagnostic difficulties. In the second patient, the mother saw abnormal development from the first month of the child's life, however this was denied by doctors.

In MRI infratentorial lesions (involving the brain stem and the cerebellum) are the early features of early-onset Krabbe disease. This disease should be suspected if the hili of the dentate nuclei, cerebellar white matter and pyramidal tracts in the brain stem are affected in an infant. No brain stem involvement was visible at the time of the first examination in either Patient 1 or Patient 2, in whom cerebellar white matter remained intact.

The first MR examination of Patient 1 was performed at the age of 6 months, before the MRI pattern fully characteristic of early-onset globoid cell leukodystrophy developed. Initially, it was concluded that observed changes were the result of an inflammatory process (cerebellitis) since in the initial assessment of this examination the supratentorial changes were ignored and only cerebellar involvement was noticed. Obviously, white matter abnormalities in the supratentorial compartment should have been noticed, however their interpretation would still be very 
Krystyna Szymańska, Agnieszka Ługowska, Milena Laure-Kamionowska, Monika Bekiesińska-Figatowska, Dorota Gieruszczak-Białek, Małgorzata Musielak, Sabrina Eichler, Anne-Katrin Giese, Arndt Rolfs

difficult. It should be emphasized here that the radiologist had no clinical information about the child. The only data in the referral sheet were as follows: "suspicion of encephalitis, protein in the cerebrospinal fluid". A vicious circle of misinformation from the parents (who claimed normal development until infection) and misunderstandings led to the diagnosis of the inflammatory process, including tuberculosis. This shows how important the flow of information between specialists is. The second brain MRI was performed 6 months after the first one. At that point the MRI pattern was already typical of GLD, although the same pattern is observed in metachromatic leukodystrophy [26]. However, the result of arylsulfatase $A$ activity analysis did not confirm this diagnosis. The radiating pattern visible on the MRI is observed in various leukodystrophies, e.g. metachromatic leukodystrophy, Krabbe disease, vanishing white matter disease. Van der Voorn tried to explain the radiating pattern in the MRI of patients with Krabbe disease [27]. They performed MR imaging in vivo and postmortem (formalin fixation) with histopathologic correlation. The areas of hyperintensity on T2weighted MR imaging corresponded to myelin-deficient foci. The authors demonstrated that many globoid cells in the periventricular regions containing lipid material, in the absence of any myelin, corresponded to the hypointense stripes on MRI and most likely contributed to shortening of both $\mathrm{T} 2$ and $\mathrm{T} 1$ values [27].

The findings in the spinal MRI in Patient 1 have already been described, at least in part: diffuse contrast enhancement of the nerve roots in the lumbar and low thoracic spine, although we also found enhancement of the meninges along the spinal cord and on the ventral surface of the medulla oblongata [9].

Brain ultrasound examination in Patient 2 revealed first abnormalities, but only an MRI of the brain suggested the possibility of Krabbe disease. This patient presented with another finding typical of Krabbe disease: thickening of the optic chiasm and prechiasmatic sections of the optic nerves. This finding is attributed to the accumulation of globoid cells and is not reported in other leukodystrophies [9,11,28].

Spectroscopic findings in Patient 2 are in accordance with the literature. Elevation of myoinositol and choline reflects demyelination, proliferation of glia and astrocytosis. Decrease in $\mathrm{N}$-acetylaspartate is caused by loss of axons [1,9].

Similar to other glycosphingolipids, the degradation of galactocerebroside in vivo requires the presence of both the enzyme $\beta$-galactocerebrosidase and its activators, namely saposins $A$ and $C$, which assist in this process. It seems that saposin $A$ is indispensable for in vivo degradation of this sphingolipid. In the clinical picture, biochemical and pathological abnormalities are qualitatively similar, but they are expressed in a milder way in SAP-A ${ }^{-/-}$mice than in twi/twi mice [18].

Originally, it was thought that saposin A deficiency does not occur in humans because the deficiency of saposin A would be compensated by saposin $C$ in vivo. Spiegel described the first case of saposin A deficit in a 6-month-old girl presenting with progressive encephalopathy [21]. She was diagnosed as suffering from $\beta$-galactocerebrosidase deficiency based on enzyme activity found in leukocytes isolated from the whole blood. The activity of $\beta$-galactocerebrosidase in skin fibroblasts was lower than in healthy controls but still within normal range. Genetic testing confirmed the presence of mutations in the prosaposin gene PSAP.

High residual activities of $\beta$-galactocerebrosidase obtained in leukocytes and fibroblasts of Patient 1 were misinformative. They were not unequivocal and it was not possible to confirm or exclude Krabbe disease in this patient. Since the MRI was not suggestive of globoid cell leukodystrophy, we supposed that Patient 1 was a compound heterozygote for a mutation causing Krabbe disease on one allele and a mutation in the saposin A domain in the PSAP gene on the second allele or he was a homozygote for mutations in the prosaposin gene. Sequencing analysis of both GALC and PSAP genes revealed two pathological mutations and some polymorphisms in the GALC gene confirming Krabbe disease in Patient 1 presented here. There are some possible explanations for the rather high residual activity of $\beta$-galactocerebrosidase found in Patient 1 . It should be kept in mind that we used an artificial fluorogenic substrate for the enzymatic analysis (6-HMU-gal). It cannot be excluded that mutations in the GALC gene result in certain changes in the $\beta$-galactocerebrosidase molecule so that the enzyme preserves the ability to hydrolyze artificial substrates. Thus, the enzymatic reaction performed in vitro does not exactly reflect the metabolic situation occurring in the affected organism. Most probably, in patients with high residual activity of $\beta$-galactocerebrosidase the enzymatic studies should also be repeated with the use of radioactive natural substrate, i.e. tritium labelled galactocerebroside, which is no longer used in our laboratory. Another explanation of high residual GALC activity is the fact that for the enzymatic analysis the whole leukocyte homogenate is used and not the purified GALC protein. It 
can be speculated that during the long incubation time (18 hr) other, not substrate-specific enzymes, present in the homogenate, can be involved in the degradation of the artificial substrate. Such an enzyme may be lysosomal $\beta$-galactosidase and its undesirable action should be specifically inhibited [17]. It should be also emphasized that enzyme activities measured in cultured skin fibroblasts are relevant to higher variability than in isolated blood leukocytes of the examined individual as the culture conditions are very changeable. For this reason, when confronted with discrepancies or doubts between biochemical results and the clinical phenotype in problematic patients, we always recommend the sequencing analysis of the GALC gene to confirm the diagnosis of Krabbe disease.

Schmidt-Sidor [20] described a foetal Krabbe disease at gestational week 20. The neuropathological examination revealed globoid cells only in some tracts in the spinal cord which showed a certain degree of myelination. No globoid cells were detected in the cerebrum or cerebellum. This is consistent with previous reports $[5,16,20]$.

In early infantile Krabbe disease the presence of globoid cells in certain structures and absence in others is linked to the myelination calendar. However, it remains unclear why certain structures like $U$ fibers are spared. It seems that it does not depend on the degree of myelination. There are probably additional factors influencing the rate of damage in particular CNS structures. This may indicate the characteristic brain MRI in adult forms of the disease with demyelination confined to the pyramidal tract. It would be interesting to check the percentage of galactocerebroside content in different neurologic tracts.

In the neuropathological picture of Patient 1 globoid cells evolved with the progress of white matter damage. Distinct areas of the brain were damaged to a varying degree. The lesion grew from the front to the rear pole of the cerebrum where it was most expressed. With the progress of white matter damage, globoid cells were more numerous and tended to cluster around blood vessels. Some of them became multinucleated. With the breakup of white matter the globoid cells were fewer in number and finally only single cells with abundant nuclei were observed.

Since the second MRI was made shortly before the death of Patient 1, it can be assumed that the changes observed in the neuropathological study correspond to those seen in the MRI as it was presented in Figures 1 and 2 .
Finally, we would like to emphasize that proper clinical-radiological-biochemical co-operation and exchange of information between parents and specialists is a key issue in the diagnosis of rare and difficult neurological diseases, particularly if the clinical picture is inconclusive.

\section{Acknowledgements}

The authors would like to thank Dr. Barbara Czartoryska for performing some of the enzymatic analyses. We are also grateful to the patients' families for their kind co-operation.

\section{References}

1. Brockmann K, Dechent P, Wilken B, Rusch O, Frahm J, Hanefeld F. Proton MRS profile of cerebral metabolic abnormalities in Krabbe disease. Neurology 2003; 60: 819-825.

2. Castelvetri LC, Givogri MI, Zhu H, Smith B, Lopez-Rosas A, Qiu X, van Breemen R, Bongarzone ER. Axonopathy is a compounding factor in the pathogenesis of Krabbe disease. Acta Neuropathol 2011; 122: 35-48.

3. Cleland WW, Kennedy EP. The enzymatic synthesis of psychosine. J Biol Chem 1960; 235: 45-51.

4. Coetzee T, Fujita N, Dupree J, Shi R, Blight A, Suzuki K, Suzuki K, Popko B. Myelination in the absence of galactocerebroside and sulfatide: normal structure with abnormal function and regional instability. Cell 1996; 86: 209-219.

5. Ellis WG, Schneider EL, McCullough JR, Suzuki K, Epstein CJ. Fetal globoid cell leukodystrophy (Krabbe disease) Pathological and biochemical examination. Arch Neurol 1973; 29: 253-257.

6. Eto Y, Suzuki K, Suzuki K. Globoid cell leukodystrophy (Krabbe's disease): Isolation of myelin with normal glycolipid composition. J Lipid Res 1970; 11: 473-479.

7. Folkerth RD, Kinney HC. Disorders of the perinatal period. Brain growth. In: Love S, Louis DN, Ellison DW (eds.). Greenfield's neuropathology. 8 ed. Hodder Arnold 2008, p. 249.

8. Galbiati F, Givogri MI, Cantuti L, Lopez Rosas A, Cao H, van Breemen R, Bongarzone ER. Combined hematopoietic and lentiviral gene-transfer therapies in newborn Twitcher mice reveal contemporaneous neurodegeneration and demyelination in Krabbe disease. J Neurosci Res 2009; 87: 1748-1759.

9. Garcia AM, Morais NM, Ohlweiler L, Winckler MI, Ranzan J, Artigalás OA, Pinto LL, Netto CB, Ashton-Prolla P, Vedolin L, Riesgo Rdos S, Rotta NT. Optic nerve enlargement and leukodystrophy: an unusual finding of the infantile form of Krabbe disease. Arq Neuropsiquiatr 2010; 68: 816-818.

10. Hagberg B, Kollberg H, Sourander P, Akesson HO. Infantile globoid cell leucodystrophy (Krabbe's disease). A clinical and genetic study of 32 Swedish cases 1953-1967. Neuropadiatrie 1969; 1: 74-88.

11. Jones BV, Barron TF, Towfighi J. Optic nerve enlargement in Krabbe's disease. Am J Neuroradiol 1999; 20: 1228-1231.

12. Kanazawa T, Nakamura S, Momoi M, Yamaji T, Takematsu H, Yano H, Sabe H, Yamamoto A, Kawasaki T, Kozutsumi Y. Inhibition 
of cytokinesis by a lipid metabolite, psychosine. J Cell Biol 2000; 149: 943-950.

13. Kozutsumi Y, Kanazawa T, Sun Y, Yamaji T, Yamamoto H, Takemat$\mathrm{su} \mathrm{H}$. Sphingolipids involved in the induction of multinuclear cell formation. Biochim Biophys Acta 2002; 1582: 138-143.

14. Krabbe KH. A new familial infantile form of diffuse brain sclerosis. Brain (Oxford) 1916; 39: 74-115.

15. Luzi P, Rafi MA, Wenger DA. Characterization of the large deletion in the GALC gene found in patients with Krabbe disease. Hum Mol Genet 1995; 4: 2335-2338.

16. Martin JJ, Leroy JG, Ceuterick C, Libert J, Dodinval P, Martin L. Fetal Krabbe leukodystrophy. A morphometric study of two cases. Acta Neuropathol 1981; 53: 87-91.

17. Martino S, Tiribuzi R, Tortori A, Conti D, Visigalli I, Lattanzi A, Biffi A, Gritti A, Orlacchio A. Specific determination of beta-galactocerebrosidase activity via competitive inhibition of beta-galactosidase. Clin Chem 2009; 55: 541-548.

18. Matsuda J, Vanier MT, Saito Y, Tohyama J, Suzuki K, Suzuki K. A mutation in the saposin A domain of the sphingolipid activator protein (prosaposin) gene results in a late-onset, chronic form of globoid cell leukodystrophy in the mouse. Hum Mol Genet 2001; 10: 1191-1199.

19. Miyatake T, Suzuki K. Globoid cell leukodystrophy: additional deficiency of psychosine galactosidase. Biochem Biophys Res Commun 1972; 48: 538-543.

20. Schmidt-Sidor B, Lewandowska E, Mierzewska H, Kleijer WJ, Czartoryska B, Wierzba-Bobrowicz T, Pasennik E. Fetal globoidal leukodystrophy (Krabbe's disease). Neuropathological picture at 20 gestational weeks. Case report (Abstr). Folia Neuropathol 1999; 37: 304 .

21. Spiegel R, Bach G, Sury V, Mengistu G, Meidan B, Shalev S, Shneor Y, Mandel H, Zeigler M. A mutation in the saposin A coding region of the prosaposin gene in an infant presenting as Krabbe disease: report of saposin A deficiency in humans. Molec Genet Metab 2005; 84: 160-166.

22. Suzuki K, Suzuki Y. Globoid cell leucodystrophy (Krabbe's disease): Deficiency of galactocerebroside p-galactosidase. Proc Natl Acad Sci USA 1970; 66: 302-309.

23. Suzuki K. Twenty five years of the "psychosine hypothesis": a personal perspective of its history and present status. Neurochem Res 1998; 23: 251-259.

24. Tappino B, Biancheri R, Mort M, Regis S, Corsolini F, Rossi A, Stroppiano M, Lualdi S, Fiumara A, Bembi B, Di Rocco M, Cooper DN, Filocamo M. Identification and characterization of 15 novel GALC gene mutations causing Krabbe disease. Hum Mutat 2010; 31: E1894-1914.

25. Tohyama J, Matsuda J, Suzuki K. Psychosine is as potent an inducer of cell death as C6-ceramide in cultured fibroblasts and in MOCH-1 cells. Neurochem Res 2001; 26: 667-671.

26. van der Knaap MS, Valk J. Globoid cell leukodystrophy (Krabbe disease). In: van der Knaap MS, Valk J. Magnetic resonance of myelination and myelin disorders. Springer-Verlag, Berlin Heidelberg 2005, pp. 91-95.

27. van der Voorn JP, Pouwels PJ, Kamphorst W, Powers JM, Lammens M, Barkhof F, van der Knaap MS. Histopathologic correlates of radial stripes on MR images in lysosomal storage disorders. AJNR Am J Neuroradiol 2005; 26: 442-446.
28. Vasconcellos E, Smith M. MRI nerve root enhancement in Krabbe disease. Pediatr Neurol 1998; 19: 151-152.

29. Wenger DA, Rafi MA, Luzi P. Molecular genetics of Krabbe disease (globoid cell leukodystrophy): diagnostic and clinical implications. Hum Mut 1997; 10: 268-279.

30. Wenger DA, Suzuki K, Suzuki Y, Suzuki K. Galactosylceramide lipidosis. Globoid cell leukodystrophy (Krabbe disease). In: Scriver CR, Beaudet AL, Sly WS, Valle D, Childs B, Vogelstein B (eds.). The Metabolic and Molecular Bases of Inherited Disease. 8 ed. McGrawHill, New York 2001, pp. 3669-3694.

31. Wenger DA. Krabbe Disease. In : Pagon RA, Bird TD, Dolan CR, Stephens K, Adam MP (eds.). Gene Reviews. University of Washington, Seattle; 2000; Update: 2011; available at: http://www.ncbi. nlm.nih.gov/books/NBK1238/\#_ncbi_dlg_citbx_NBK1238.

32. White AB, Givogri MI, Lopez-Rosas A, Cao H, van Breemen R, Thinakaran G, Bongarzone ER. Psychosine accumulates in membrane microdomains in the brain of Krabbe patients, disrupting the raft architecture. J Neurosci 2009; 29: 6068-6077. 